\title{
Comparação das Técnicas Transarterial e de Estimulação de Múltiplos Nervos para Bloqueio do Plexo Braquial por Via Axilar usando Lidocaína com Epinefrina *
}

\section{Comparison of Transarterial and Multiple Nerve Stimulation Techniques for Axillary Block using Lidocaine with Epinephrine}

\author{
Luiz Eduardo Imbelloni, TSA ${ }^{1}$; Lúcia Beato, TSA ${ }^{2}$; José Antônio Cordeiro ${ }^{3}$
}

\begin{abstract}
RESUMO
Imbelloni LE, Beato L, Cordeiro JA - Comparação das Técnicas Transarterial e de Estimulação de Múltiplos Nervos para Bloqueio do Plexo Braquial por Via Axilar usando Lidocaína com Epinefrina
\end{abstract}

JUSTIFICATIVA E OBJETIVOS: A técnica transarterial com grandes doses de anestésico local resulta em alta efetividade para o bloqueio axilar do plexo braquial. A técnica utilizando múltiplos estímulos exige mais tempo e maior experiência. Este estudo prospectivo compara a latência e o índice de sucesso do bloqueio do plexo braquial usando duas técnicas de localização: transarterial ou múltipla estimulação dos nervos.

MÉTODO: A lidocaína com epinefrina, $800 \mathrm{mg}$, foi usada inicialmente para o bloqueio axilar. No grupo transarterial, 30 $\mathrm{mL}$ de lidocaína a 1,6\% com epinefrina foram injetados profundamente e $20 \mathrm{~mL}$ superficialmente à artéria axilar. No grupo de múltipla estimulação, três nervos foram localizados eletricamente e bloqueados com volumes $20 \mathrm{~mL}, 20 \mathrm{~mL}$ e 10 $m L$ da solução. O bloqueio foi considerado efetivo quando a analgesia estava presente em todos os nervos na área distal ao cotovelo.

RESULTADOS: O tempo de latência ( $8,8 \pm 2,3$ min versus 10,2 $\pm 2,4$ min; $p$-valor $=0,010)$ foi significativamente menor no grupo transarterial. Bloqueios sensitivos completos nos quatro nervos (mediano, ulnar, radial e musculocutâneo) foram obtidos em $92,5 \%$ versus $83,3 \%$ no grupo de múltipla estimulação e acesso transarterial, respectivamente sem diferença significativa $(p$-valor $=0,68)$. O nervo musculocutâneo foi significativamente mais fácil de bloquear com o estimulador de nervo periférico $(p=0,034)$.

CONCLUSÕES: A técnica de múltipla estimulação para o bloqueio axilar usando estimulador de nervos (3 injeções) e a

\footnotetext{
* Recebido do (Received from) Instituto de Anestesia Regional, São José do Rio Preto, SP

1. Diretor do Instituto de Anestesia Regional do Hospital de Base de São José do Rio Preto, SP; Anestesiologista da Clínica São Bernardo e Casa de Saúde Santa Maria. Rio de Janeiro, RJ

2. Anestesiologista da Clínica São Bernardo e Casa de Saúde Santa Maria. Rio de Janeiro

3. Professor na Faculdade de Medicina de São José do Rio Preto,
} FAMERP, São José do Rio Preto, SP

Apresentado (Submitted) em 23 de junho de 2004

Aceito (Accepted) para publicação em 25 de outubro de 2004

Endereço para correspondência (Correspondence to)

Dr. Luiz Eduardo Imbelloni

Av. Epitácio Pessoa, 2356/203 Lagoa

22471-000 Rio de Janeiro, RJ

E-mail:dr.imbelloni@terra.com.br

(C) Sociedade Brasileira de Anestesiologia, 2005 técnica transarterial (2 injeções) produzem resultados semelhantes na qualidade do bloqueio. O nervo musculocutâneo é mais facilmente bloqueado com o uso do estimulador de nervo periférico. A técnica de múltipla estimulação necessitou menor suplementação do bloqueio e aumentou o tempo para o início da cirurgia.

Unitermos: ANESTÉSICOS, Local: epinefrina, lidocaína; TÉCNICAS ANESTÉSICAS, Regional: bloqueio axilar, plexo braquial

\section{SUMMARY}

Imbelloni LE, Beato L, Cordeiro JA - Comparison of Transarterial and Multiple Nerve Stimulation Techniques for Axillary Block using Lidocaine with Epinephrine

BACKGROUND AND OBJECTIVES: High-dose transarterial technique results in highly effective axillary block. The multiple nerve stimulation technique (MNS) requires more time and experience. This prospective study aimed at comparing onset and success rate of multiple-injection axillary brachial plexus block using two methods of nerve location: transarterial or multiple nerve stimulation technique.

METHODS: Axillary block was initially induced with $800 \mathrm{mg}$ lidocaine with epinephrine. The transarterial group received deeply injected $30 \mathrm{~mL}$ of $1.6 \%$ lidocaine with epinephrine, and $20 \mathrm{~mL}$ superficially to the axillary artery. For the multiple nerve stimulation group, three terminal motor nerves were electrolocated and blocked with $20 \mathrm{~mL}, 20 \mathrm{~mL}$ and $10 \mathrm{~mL}$. Blockade was considered effective when analgesia was present in all sensory nerves distal to the elbow.

RESULTS: Onset $(8.8 \pm 2.3 \mathrm{~min}$ versus $10.2 \pm 2.4 \mathrm{~min}$; $p$-value $=0.010$ ) was significantly shorter in the transarterial group. Complete sensory block of all four nerves (median, ulnar, radial and musculocutaneus) was achieved in $92.5 \%$ versus $83.3 \%$ for multiple nerve stimulation group and transarterial group, respectively, without significant difference ( $p=0.68)$. Musculocutaneous nerve was significantly easier to be blocked with the aid of peripheral nerve stimulator $(p=0.034)$.

CONCLUSIONS: Both MNS technique for axillary block with nerve stimulator (3 injections) and transarterial technique (2 injections) promote similar results. Musculocutaneous nerve is more easily blocked with the aid of peripheral nerve stimulator. MNS technique has required less supplementary blocks and has delayed beginning of surgery.

Key Words: ANESTHETIC, Local: epinephrine, lidocaine; ANESTHETIC TECHNIQUES, Regional: axillary block, brachial plexus

\section{INTRODUÇÃO}

plexo braquial pode ser localizado de diversas formas, incluindo o uso de parestesia, punção transarterial, perda da resistência na bainha nervosa e neuroestimulação. A via axilar tem sido indicada para procedimentos cirúr- 
gicos no antebraço e mão, não se excluindo também procedimentos no braço ${ }^{1-3}$. A técnica de estimulação múltipla no bloqueio axilar, em que cada nervo é localizado com estimulador e as injeções realizadas separadamente, proporciona um alto índice de sucesso e diminui o tempo de instalação $^{4-7}$.

A axila é uma região com pequeno risco de lesão de estruturas vitais, sendo a artéria axilar um excelente guia para execução do bloqueio axilar. A técnica transarterial é executada com a deposição dos anestésicos locais em duas áreas separadas pela artéria axilar, apresentando baixa incidência de falhas ${ }^{1,5}$. A neuroestimulação permite identificar com facilidade cada uma das quatro respostas motoras possíveis: dos nervos mediano, ulnar e radial, estimulados no interior da bainha nervosa e do nervo musculocutâneo, na parte alta da axila ou perto do músculo córaco-braquial. Desta forma, a neuroestimulação permite a injeção fracionada do anestésico local de modo dirigido e, diferentemente da técnica da busca de parestesias com a agulha, apresenta baixa probabilidade de traumatismo nervoso direto. Diversos trabalhos de bloqueio individualizado do plexo braquial demonstraram que a extensão e a profundidade da anestesia do membro superior era melhor com localização e bloqueio dos 4 ramos terminais, apesar de maior consumo de tempo para realização do procedimento que se compensava com menor período de latência $^{4-8}$.

O objetivo deste estudo prospectivo é comparar a latência, tempo de execução, efetividade e falhas do bloqueio do plexo braquial obtida com as duas técnicas de identificação da presença da agulha no compartimento perivascular axilar: estimulação múltipla e injeção perivascular transarterial.

\section{MÉTODO}

Após aprovação pela Diretoria de Publicação e Divulgação da Clínica ligada à Comissão de Ética, participaram deste estudo prospectivo 70 pacientes com idade entre 20 e 60 anos, estado físico ASA I e II, submetidos a cirurgias ortopédicas de antebraço e mão, divididos aleatoriamente em dois grupos (grupo MEN = 40 pacientes e grupo TA $=30$ pacientes), de acordo com a técnica utilizada. Todos os pacientes receberam informações detalhadas sobre a técnica a que se submeteriam, após terem dado consentimento.

Nenhum paciente recebeu medicação pré-anestésica. Após venóclise com cateter $18 \mathrm{G}$ ou $20 \mathrm{G}$, foi iniciada infusão lenta de solução de Ringer com lactato. A monitorização na sala de operações foi realizada pelo ECG contínuo na posição CM5, pressão arterial por método não-invasivo e oximetria de pulso. Administraram-se inicialmente, por via venosa, 50 a 100 $\mu \mathrm{g}$ de fentanil associados a $10 \mathrm{mg}$ de metoclopramida. Os pacientes só receberam sedação com midazolam após instalação do bloqueio e início da cirurgia.

\section{Técnica de Múltiplos Estímulos (MEN)}

Os pacientes foram colocados em decúbito dorsal com o braço abduzido $90^{\circ}$ e o antebraço estendido ou fletido. Aartéria axilar foi desenhada no sulco do músculo bíceps, na linha que une a inserção umeral dos músculos peitoral e grande dorsal. Marcou-se um ponto $3 \mathrm{~cm}$ abaixo onde foi realizada infiltração subcutânea anterior e posterior à artéria para a entrada da agulha conectada a um estimulador. Após o botão dérmico no ponto marcado, uma agulha B. Braun ${ }^{\circledR} 22 \mathrm{G} 0,70 x$ $50 \mathrm{~mm}$, eletricamente isolada, foi conectada a um estimulador de nervo periférico (Stimuplex ${ }^{\circledR} \mathrm{R}$, B. Braun Melsungen $A G)$ regulado para liberar uma corrente pulsátil quadrada de $1 \mathrm{~mA}$, com intensidade reduzida até $0,5 \mathrm{~mA}$, e inserida num ângulo de $30^{\circ} \mathrm{com}$ a pele, na direção da região posterior à artéria axilar. Buscou-se estimular o nervo ulnar, com resposta dos músculos da eminência tenar e hipotenar e pinçamento dos dedos mínimo e polegar, ou o nervo radial, com extensão da mão. Injetaram-se $20 \mathrm{~mL}$ de lidocaína a 1,6\% com epinefrina 1:200.000, preparada na hora. Aagulha foi então retirada e re-inserida anteriormente à artéria axilar, procurando-se estimular o nervo mediano e obter contrações musculares da face anterior do antebraço e flexão da mão. Injetaram-se nesse ponto mais $20 \mathrm{~mL}$ da solução preparada. Aseguir, a agulha foi retirada do feixe axilar e procurou-se o nervo musculocutâneo, orientando-a no sentido da apófise coracóide, na inserção do músculo córaco-braquial. Como a obtenção de contração do músculo bíceps e flexão do antebraço sobre o braço, injetaram-se $10 \mathrm{~mL}$ da solução preparada. Em 5 pacientes aleatoriamente selecionados foi injetado 1 $\mathrm{mL}$ de contraste iohexol ( $\left.300 \mathrm{mg} \cdot \mathrm{mL}^{-1}\right)$ em cada nervo, antes da injeção do anestésico para estudo radiológico.

\section{Técnica Transarterial (TA)}

Os pacientes foram colocados em posição supina, com o braço em abdução de $90^{\circ}$, o cotovelo flexionado e a mão sob a cabeça. A artéria axilar foi palpada e marcada. Uma agulha hipodérmica calibre $30 \times 8$ dirigida perpendicularmente à artéria, foi introduzida lentamente até o refluxo de sangue, sendo então introduzida um pouco além até a transfixação da mesma, confirmada pela interrupção desse refluxo. Nesse momento, $30 \mathrm{~mL}$ de lidocaína a 1,6\% com epinefrina $1: 200.000$, preparada na hora, foram injetados lentamente, observando-se possíveis sinais de injeção vascular. A aguIha foi então recuada lentamente passando mais uma vez pelo lúmen da artéria e posicionada anteriormente a ela, onde foram injetados os restantes $20 \mathrm{~mL}$ da solução. A aguIha era então removida e o braço colocado ao longo do corpo com compressão do oco axilar. Em 5 pacientes aleatoriamente selecionados, foi injetado $1 \mathrm{~mL}$ de contraste iohexol (300 mg. $\mathrm{mL}^{-1}$ ) em cada lado da artéria, antes da injeção do anestésico para estudo radiológico.

Aanalgesia foi avaliada em ambos os grupos por pinçamento da pele, observando-se manifestações do paciente quanto à sensibilidade para dor, com o objetivo de determinar a extensão do bloqueio sensitivo dos nervos musculocutâneo, na borda radial do antebraço, radial, na face dorsal da mão, ulnar, na parte medial da mão e dedo mínimo e do mediano, na palma da mão. Foram avaliados os seguintes parâmetros: 1) latência da anestesia: tempo decorrido do início da punção 
até a perda da sensibilidade nas áreas dos quatro nervos; 2) duração do bloqueio sensitivo: tempo entre o início da punção e a completa recuperação da sensibilidade; 3) duração do bloqueio motor: tempo entre o início da punção e a completa recuperação dos movimentos do membro bloqueado; 4) percepção do garrote e 5) complicações e efeitos colaterais.

O bloqueio foi considerado completo se todos os nervos fossem bloqueados com o volume preconizado. Se uma injeção suplementar fosse necessária ou houvesse necessidade de complementação da anestesia, o bloqueio era considerado incompleto. Neste caso, a dose suplementar com anestésico envolveria apenas o nervo da área não bloqueada. Considerou-se falha de bloqueio à necessidade de anestesia geral.

Durante a injeção do anestésico, sinais de toxicidade da lidocaína foram pesquisados, como zumbido, gosto metálico, sensação de calor, distúrbios visuais, tontura ou abalos musculares.

Ao final da cirurgia, foram administrados tenoxicam $(40 \mathrm{mg}) \mathrm{e}$ dipirona (30 mg. $\mathrm{kg}^{-1}$ ) em $100 \mathrm{~mL}$ de Ringer com lactato, sendo então o paciente transferido para o quarto. Por 48 horas, todos eles foram acompanhados por telefone para verificação de complicações no local do bloqueio. Se houvesse algum indício, o paciente retornaria ao hospital onde seria avaliado pelos autores. Todos os pacientes foram questionados quanto à satisfação com a técnica.

Os resultados foram avaliados utilizando o teste t com correção de Welch para os graus de liberdade e comparações de dois percentuais pelo teste exato de Fisher com nível de significância para $p<0,05$.

\section{RESULTADOS}

Os resultados de variáveis contínuas estão apresentados como: média \pm desvio-padrão. A distribuição da idade, peso, altura, sexo estão na tabela I; tempo de latência e duração dos bloqueios sensitivo e motor estão na tabela II. O estudo contrastado de cada nervo com a técnica de múltiplos estímulos está retratado nas figuras 1,2 e 3, e com a técnica transarterial nas figuras 4 e 5 .
Tabela I - Dados Demográficos

\begin{tabular}{lccc}
\hline & $\begin{array}{c}\text { Grupo MEN } \\
\mathrm{n}=40\end{array}$ & $\begin{array}{c}\text { Grupo TA } \\
\mathrm{n}=30\end{array}$ & Valor de $\mathrm{p}$ \\
\hline Idade $(\mathrm{anos}){ }^{*}$ & $38,6 \pm 12$ & $36,9 \pm 10,6$ & 0,53 \\
Peso $(\mathrm{kg})^{*}$ & $67,7 \pm 11,4$ & $71,2 \pm 12,4$ & 0,13 \\
Altura $(\mathrm{cm}){ }^{*}$ & $166,4 \pm 9,3$ & $168,6 \pm 10$ & 0,35 \\
Sexo & & & \\
$\quad$ Masculino & 22 & 17 & \\
$\quad$ Feminino & 18 & 13 & \\
\hline
\end{tabular}

Valores expressos pela Média \pm DP

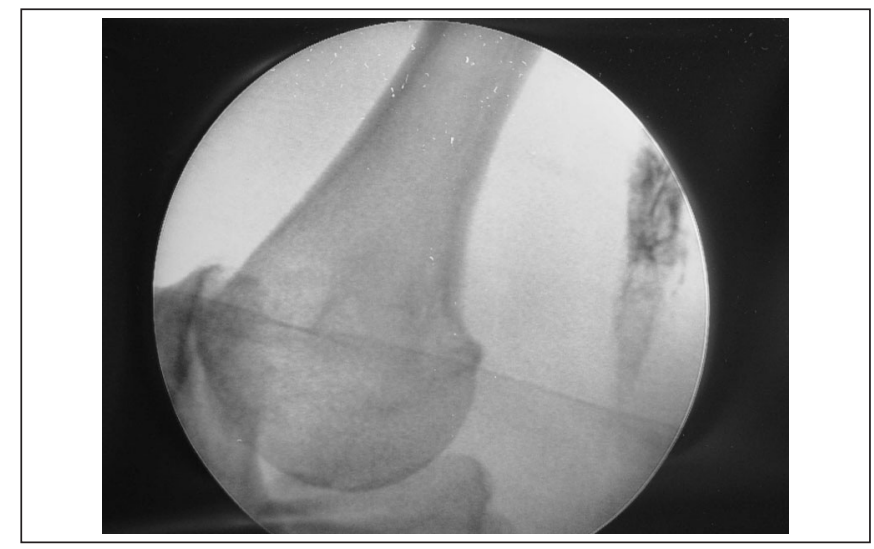

Figura 1 - Nervo Mediano Contrastado com Estimulador de Nervos

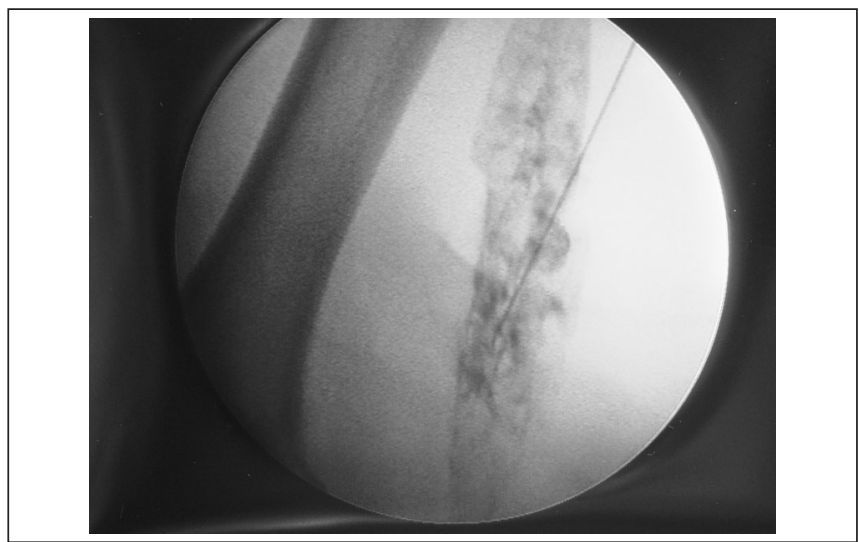

Figura 2 - Nervo Radial Contrastado com Estimulador de Nervos

Tabela II - Dados Anestésicos

\begin{tabular}{lccc}
\hline & $\begin{array}{c}\text { Grupo MEN } \\
\mathrm{n}=40\end{array}$ & $\begin{array}{c}\text { Grupo TA } \\
\mathrm{n}=30\end{array}$ & Valor de $\mathrm{p}$ \\
\hline Dose média de lidocaína $\left(\mathrm{mg} \cdot \mathrm{kg}^{-1}\right)$ & 13,5 & 12,5 & 15,09 \\
Dose máxima de lidocaína $\left(\mathrm{mg}_{\mathrm{kg}} \mathrm{k}^{-1}\right)$ & 16,6 & $8,8 \pm 2,3$ & 0,010 \\
Tempo de latência $(\mathrm{min})$ & $10,2 \pm 2,4$ & $184,3 \pm 26,1$ & 0,022 \\
Duração bloqueio sensitivo $(\mathrm{min})$ & $198,4 \pm 25,8$ & $188 \pm 31,9$ & 0,038 \\
Duração bloqueio motor $(\mathrm{min})$ & $201,4 \pm 20,2$ & 29 & 0,89 \\
Satisfação com a técnica $(\mathrm{n})$ & 40 & & \\
\hline
\end{tabular}




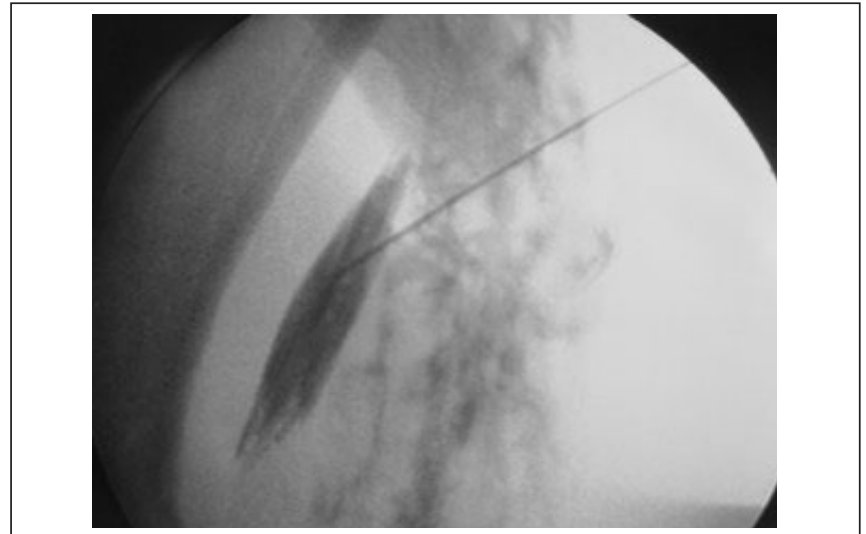

Figura 3 - Nervo Musculocutâneo Contrastado por Estimulador de Nervos

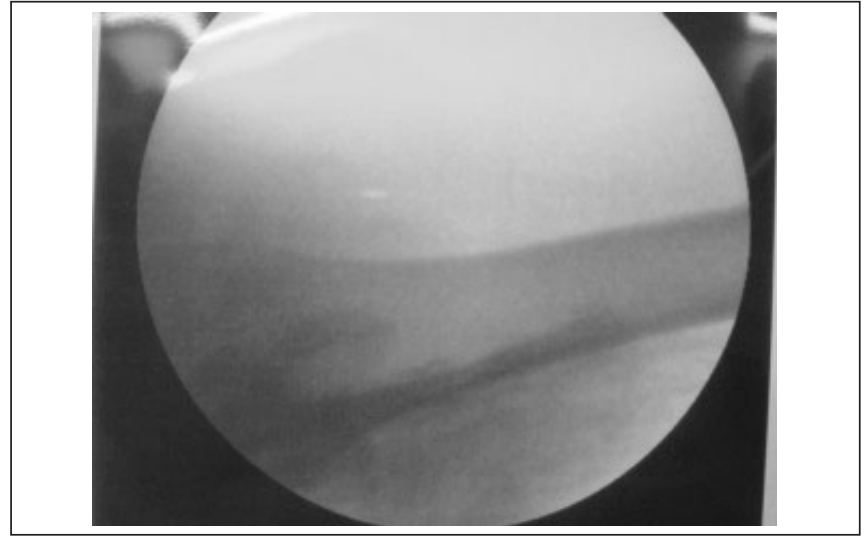

Figura 4 - Contraste por Injeção Posterior à Artéria Axilar

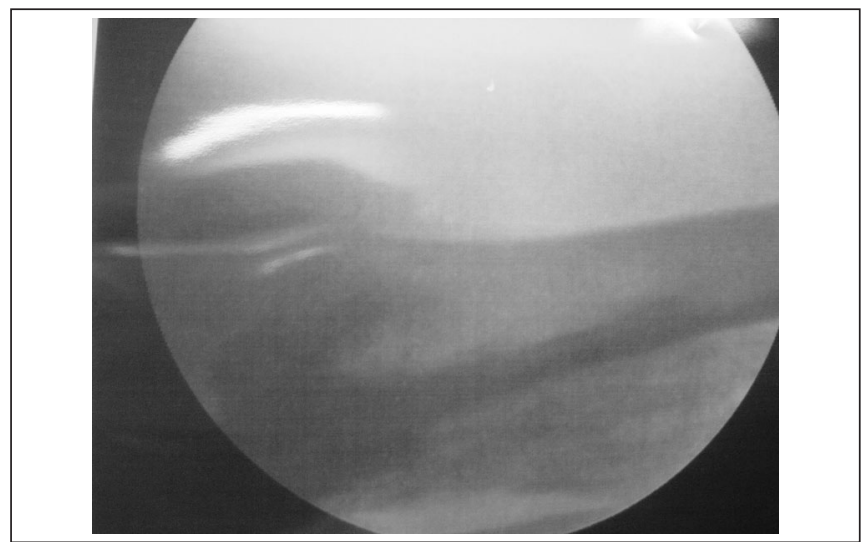

Figura 5 - Contraste por Injeção Anterior à Artéria Axilar

Em relação ao tempo médio de latência, observa-se que foi significativamente menor no grupo TA. Durante a realização da técnica com múltiplos estímulos, não houve qualquer caso de punção da artéria ou da veia.
Apenas dois pacientes, ambos do grupo TA, necessitaram de anestesia geral; todos os demais foram operados com os bloqueios. Em todos pacientes do grupo TA a artéria foi transfixada e o anestésico depositado posterior e anteriormente a ela. No grupo MEN, obteve-se contração muscular nos territórios inervados pelo mediano, radial ou ulnar e musculocutâneo em todos os pacientes. Houve necessidade de complementação em dois pacientes no grupo MEN e em três pacientes no grupo TA(Tabela II). O índice de sucesso de $92,5 \%$ com MEN foi semelhante aos $83,3 \%$ do grupo TA. O nervo musculocutâneo foi significativamente mais fácil de ser bloqueado com uso do estimulador de nervos $(p=0,034)$ (Tabela III).

Tabela III - Nervos Bloqueados, Complementação dos Nervos e Qualidade da Analgesia. Número de Pacientes em Cada Grupo

\begin{tabular}{lccc}
\hline & $\begin{array}{c}\text { Grupo MEN } \\
\mathrm{n}=40\end{array}$ & $\begin{array}{c}\text { Grupo TA } \\
\mathrm{n}=30\end{array}$ & Valor de $\mathrm{p}$ \\
\hline Nervos Bloqueados & 39 & 28 & 0,12 \\
$\quad$ Radial & 39 & 29 & 1,0 \\
$\quad$ Ulnar & 40 & 29 & 0,43 \\
$\quad$ Mediano & 39 & 25 & 0,034 \\
$\quad$ Musculocutâneo & & & 0,12 \\
Complementação dos Nervos & 1 & 0 & \\
$\quad$ Radial & 1 & 0 & \\
$\quad$ Ulnar & 0 & 0 & \\
$\quad$ Mediano & 1 & 3 & \\
$\quad$ Musculocutâneo & $(92,5 \%)$ & $(83,3 \%)$ & 0,68 \\
Qualidade da Analgesia & 37 & 25 & \\
Adequada & 3 & 3 & \\
Parcial & 0 & 2 & \\
Falha (anestesia geral) & & & \\
\hline & &
\end{tabular}

A duração dos bloqueios sensitivo e motor foi significativamente maior no grupo MEN em comparação com o TA. A dose média de lidocaína foi de $13,5 \mathrm{mg} \cdot \mathrm{kg}^{-1}$ no grupo MEN, com maior dose de $16,6 \mathrm{mg} \cdot \mathrm{kg}^{-1}$. No grupo TA foi de 12,5 $\mathrm{mg} \cdot \mathrm{kg}^{-1}$, com dose máxima de $15,09 \mathrm{mg} \cdot \mathrm{kg}^{-1}$. Em ambos os grupos, não se verificou qualquer sinal clínico de toxicidade sistêmica do anestésico local.

Nenhum paciente referiu dor no local da cirurgia. Entretanto, quatro pacientes relataram dor ao torniquete sendo dois no grupo TAe dois no grupo MEN, sem diferença significante.

Não foram observadas lesões nervosas no grupo MEN e tampouco hematoma ou injeção vascular do anestésico no grupo TA.

\section{DISCUSSÃo}

Quando várias injeções de lidocaína com epinefrina são aplicadas no plexo braquial via axilar, o uso do estimulador de plexo (3 injeções) resulta numa incidência de sucesso comparável com a técnica transarterial (2 injeções), demandando porém, maior tempo para início da cirurgia. Diversos auto- 
res consideram como sucesso do bloqueio axilar a presença de analgesia em todas as áreas de abrangência da cirurgia. Esta definição é suficiente do ponto de vista clínico, mas implica em um falso índice de sucesso, quando comparado com diferentes técnicas de bloqueio ${ }^{5}$. Para este trabalho, o bloqueio foi considerado completo quando todos os nervos do antebraço foram bloqueados com a primeira injeção. No grupo dos múltiplos estímulos nervosos, foi necessária complementação em três pacientes e em diferentes nervos: musculocutâneo, radial e ulnar. No grupo da punção transarterial, houve necessidade de complementação do musculocutâneo em três pacientes e falha do bloqueio em outros dois pacientes.

Quando se realiza o bloqueio do plexo braquial, existe uma preocupação constante com o volume a ser injetado. A relação entre o volume de anestésico injetado e a extensão da analgesia foi primeiramente descrita em $1961^{9}$. Embora o cálculo do volume do compartimento neurovascular axilar seja em média de apenas $42 \mathrm{~mL}^{9}$, a injeção única perivascular, usando entre 60 a $80 \mathrm{~mL}$, pode resultar em bloqueios parciais ${ }^{10,11}$. Isto foi inicialmente explicado pela dispersão proximal insuficiente do anestésico para os ramos nervosos e divisões superiores do plexo causada pela cabeça do úmero, mas não confirmada por estudos aleatoriamente controla$\operatorname{dos}^{12,13}$.

Duas técnicas do bloqueio axilar melhoram a distribuição do anestésico dentro da bainha neurovascular, quando comparadas com técnica de injeção única: a transarterial, constituindo-se numa dupla injeção, e os múltiplos estímulos nervosos, que pode ser uma técnica de injeção tripla ou quádrupla. A técnica transarterial com $800 \mathrm{mg}$ de lidocaína a 1,6\% com epinefrina resultou num índice de sucesso de $95 \%$ a $100 \%$, sem qualquer efeito adverso ${ }^{1}$. Estudos anatômicos mostraram que os nervos musculocutâneo e axilar deixam a bainha neurovascular na altura do processo coracóide, em concordância com o fato de que a injeção de $50 \mathrm{~mL}$ de lidocaína a $1,6 \%$ não bloqueou o nervo musculocutâneo em três pacientes desta série. Existem poucos estudos com a técnica de múltiplos estímulos com estimulador de nervo. Uma comparação de três técnicas de bloqueio axilar (cateter, parestesia e nervo estimulação) mostrou que há uma tendência de maior sucesso se mais de um nervo é estimulado e a injeção realizada (50\% para 1, $80 \%$ para 2 e $100 \%$ para 3 locais de estímulos) ${ }^{14}$. A técnica de três estímulos, quando apenas os nervos mediano, radial e ulnar são localizados, produz um índice de sucesso de apenas $50 \%$ no bloqueio axilar ${ }^{15}$. Neste estudo foi incluído o estímulo isolado do musculocutâneo resultando numa incidência de $92,5 \%$ de sucesso.

Quando a solução de anestésico foi injetada perto de 2 ou 4 nervos identificados, apenas 1 em 15 pacientes necessitaram suplementação ${ }^{15}$. A comparação entre uma injeção simples e a injeção após localização de 3 nervos resultou numa efetividade de $43 \%$ versus $90 \%$, respectivamente ${ }^{4}$. Recentemente foi demonstrado que a dupla estimulação resulta em $92 \%$ de sucesso, enquanto que a injeção única alcançou um índice de apenas $52 \%{ }^{16}$. Nesse estudo, comparando a tripla injeção após localização de três nervos com neuroestimula- dor com a técnica de duas injeções pela via transarterial, verificou-se um índice de sucesso de $92,5 \%$ com a tripla injeção comparado com 83,3\% da dupla injeção.

O índice de sucesso depende do local da cirurgia e da extensão do bloqueio ${ }^{17}$ sendo, portanto, difícil compará-lo entre diferentes estudos. No presente trabalho, a preocupação maior foi com a dispersão da analgesia e menor com o bloqueio motor para o sucesso da cirurgia. Inicialmente não se procurou o nervo que inervava a área operada, mas sim um estímulo interno e externo à artéria axilar. O nervo mediano é freqüentemente mais fácil de se localizar quando se utiliza o estimulador de nervos, e sua pesquisa tornou-se popular para o método de injeção única ${ }^{17}$.

O bloqueio incompleto do nervo musculocutâneo é um problema comum na técnica axilar, pois ele não está próximo da artéria axilar ${ }^{18}$. Neste estudo, a dupla injeção com a técnica transarterial e a procura do estímulo deste nervo na técnica dos três estímulos demonstrou que este problema pode ser minimizado com esta última abordagem.

O acesso transarterial é realizado com dupla injeção, sendo uma superficial e outra profunda à artéria axilar. $O$ índice de sucesso de $83,3 \%$ neste estudo, ficou próximo ao observado em alguns trabalhos $\left(79 \%{ }^{19}, 88,8 \%{ }^{20}\right)$, porém menor do que os $92 \%{ }^{16}$ e $95 \%{ }^{1}$ de outros. Já o índice de sucesso de $92,5 \%$ verificado aqui com a utilização de múltiplos estímulos é semelhante aos obtidos em outros estudos, que variaram de $88 \%$ a $93 \%{ }^{4,5,8}$.

Apesar do aumento do risco de complicações como vasoespasmo, hematoma e/ou pseudoaneurisma ${ }^{21,22}$, a técnica da injeção transarterial é considerada segura, o que demonstra uma análise prospectiva de 1000 pacientes ${ }^{20}$. Neste estudo, o acompanhamento por 48 horas no pós-operatório não evidenciou qualquer complicação. A maior preocupação durante a localização com múltiplos estímulos é a possibilidade de neuropraxias causadas pela agulha de punção. Esta possibilidade foi reduzida pelo uso do estimulador de nervo, assim como pelo avanço lento da agulha enquanto a corrente era reduzida até $0,5 \mathrm{~mA}$. Além disso, a suplementação da analgesia nunca foi realizada no mesmo local da injeção anterior.

A dose máxima de lidocaína com epinefrina recomendada para anestesia regional em adultos é de $7 \mathrm{mg} \cdot \mathrm{kg}^{-1} 23$. Entretanto, quando a lidocaína é usada diluída e em anestesia infiltrativa, a dose varia de $45 \mathrm{mg} \cdot \mathrm{kg}^{-1} \mathrm{em}$ pacientes magros a 50 $\mathrm{mg} \cdot \mathrm{kg}^{-1}$ em pacientes obesos ${ }^{24}$. A dose de lidocaína para se obter concentração plasmática de $5 \mu \mathrm{g} \cdot \mathrm{mL}^{-1}$ varia com o local de administração ${ }^{25}$. Em 346 pacientes nos quais se utilizaram $900 \mathrm{mg}$ de lidocaína com epinefrina $(30 \mathrm{~mL}$ a $2 \%$ e $30 \mathrm{~mL}$ a $1 \%$ ) para bloqueio do plexo braquial por via transarterial, não se observou qualquer sinal de intoxicação e o índice de sucesso foi de $100 \%{ }^{26}$. Outro estudo encontrou um valor médio de concentração plasmática após bloqueio de plexo braquial por via transarterial com 900 mg de lidocaína de 2,9 $\mu \mathrm{g} \cdot \mathrm{mL}^{-1}$, com maior valor individual de $5,6 \mu \mathrm{g} \cdot \mathrm{mL}^{-1}$, representando uma dose de $18 \mathrm{mg} \cdot \mathrm{kg}^{-1} 27$. A dose de $900 \mathrm{mg}$ utilizada rotineiramente em mais de 500 pacientes pela via transarterial $^{27}$ é $12,5 \%$ maior do que os 800 mg utilizados no presente Vol. 55, No 1, Janeiro - Fevereiro, 2005 
trabalho. Amaior dose utilizada foi de $16,6 \mathrm{mg} \cdot \mathrm{kg}^{-1}$, menor do que os $18 \mathrm{mg} \cdot \mathrm{kg}^{-1}$ utilizados no referido trabalho ${ }^{27}$. Da mesma forma, não se observaram manifestações clínicas de toxicidade sistêmica com a lidocaína com epinefrina.

Não se observaram, nessa série, pacientes ansiosos com a realização de múltiplas injeções, provavelmente devido à explicação prévia do método, à anestesia do trajeto da agulha, à estimulação com amperagem de 0,5 a $1 \mathrm{~mA}$ e ao uso prévio de fentanil.

Concluindo, quando o bloqueio do plexo braquial via axilar é realizado com ajuda do estimulador de nervo periférico e o nervo musculocutâneo é estimulado juntamente com o radial e/ou mediano e/ou ulnar, o resultado é um bloqueio semeIhante ao obtido através de dupla injeção pela técnica transarterial, porém com maior efetividade no bloqueio do musculocutâneo. No entanto, o tempo para permitir o início da cirurgia é maior com uso do neuroestimulador.

\section{Comparison of Transarterial and Multiple Nerve Stimulation Techniques for Axillary Block using Lidocaine with Epinephrine}

Luiz Eduardo Imbelloni, TSA, M.D.; Lúcia Beato, TSA, M.D.; José Antônio Cordeiro, M.D.

\section{INTRODUCTION}

Brachial plexus may be located in different ways, including paresthesia, transarterial puncture, loss of nervous sheath resistance and neurostimulation. Axillary route has been indicated for forearm, arm and hand procedures ${ }^{1-3}$. The multiple stimulation technique during axillary block, in which each nerve is located with stimulator and injections are performed separately, provides high success rates and decreases installation time ${ }^{4-7}$.

The axilla is a region at low risk of vital structures damage, being the axillary artery an excellent guide for axillary block. The transarterial technique is performed by injecting local anesthetics in two different areas separated by the axillary artery and has low incidence of failures ${ }^{1,5}$. Neurostimulation allows the easy identification of each of the four possible motor responses: median, ulnar and radial nerves stimulated within the nervous sheath, and musculocutaneous nerve in the upper axilla or close to coracobrachial muscle. This way, neurostimulation allows for fractional and directed local anesthetic injection and, differently from paresthesia search with a needle, has a low probability of direct nervous trauma. Several studies on individualized brachial plexus block have shown that upper limb anesthetic extension and depth were better with location and blockade of the 4 terminal branches, notwithstanding the longer time for the procedure, which would be balanced by faster onset ${ }^{4-8}$.
This prospective study aimed at comparing onset, duration, effectiveness and failures of brachial plexus block induced with two techniques to identify the presence of the needle in the axillary perivascular compartment: multiple stimulation and transarterial perivascular injection.

\section{METHODS}

After the Publication and Disclosure Board of Directors approval, participated in this prospective study 70 patients aged 20 to 60 years, physical status ASA I and II, submitted to orthopedic forearm and hand surgeries who were randomly distributed in two groups (group MNS $=40$ patients and group TA $=30$ patients) according to the technique. All patients gave their informed consent after being informed in detail about the technique.

Patients were not premedicated. After venoclysis with $18 \mathrm{G}$ or $20 \mathrm{G}$ catheter, lactated Ringer's solution was slowly injected. Monitoring in the operating room consisted of continuous ECG at CM5, noninvasive blood pressure and pulse oximetry. The patients received 50 to $100 \mu \mathrm{g}$ intravenous fentanyl associated to $10 \mathrm{mg}$ metochlopramide and were only sedated with midazolam after blockade installation and beginning of surgery.

\section{Multiple Stimulation Technique (MNS)}

Patients were placed in the supine position with $90^{\circ} \mathrm{arm}$ abduction and forearm extended or flexed. Axillary artery was drawn in the biceps muscle sulcus, on the line connecting the umeral insertion of pectoral and greater dorsal muscles. A point was marked $3 \mathrm{~cm}$ below this, were infiltration was performed anterior and posterior to the artery for the entry of the needle connected to the stimulator.

After a skin wheal raised on the marked point, a B. Braun 22G $0.70 \times 50 \mathrm{~mm}$ electrically isolated needle was connected to a peripheral nerve stimulator (Stimuplex ${ }^{\circledR} \mathrm{R}$, B. Braun Melsungen $A G$ ) adjusted to release a pulsatile square $1 \mathrm{~mA}$ current with intensity decreased to $0.5 \mathrm{~mA}$ and inserted in $30^{\circ}$ angle with the skin posterior to the axillary artery. We tried to stimulate the ulnar nerve with responses of thenar and hypothenar eminence muscles and clamping of fifth finger and thumb, or the radial nerve with hand extension.

Recently prepared $20 \mathrm{~mL}$ of $1.6 \%$ lidocaine with $1: 200,000$ epinephrine were injected. Needle was then removed and reinserted anterior to the axillary artery, in an attempt to stimulate the median nerve and obtain muscle contractions of anterior forearm and hand flexion. At this point, additional $20 \mathrm{~mL}$ of the prepared solution were injected. After, needle was removed from the axillary bundle and musculocutaneous nerve was looked for by orienting the needle toward the coracoid apophysis at the insertion of the coracobrachial muscle. When biceps contraction and flexion of the forearm over the arm were reached, $10 \mathrm{~mL}$ of the prepared solution were injected. 
Five randomly selected patients received $1 \mathrm{~mL}$ iohexol contrast $\left(300 \mathrm{mg}_{\mathrm{mL}}{ }^{-1}\right)$ in each nerve before anesthetic injection for radiological evaluation.

Transarterial Technique (TA)

Patients were placed in the supine position with $90^{\circ}$ arm abduction, flexed elbow and hand under the head. Axillary artery was palpated and marked. A $30 \times 8$ needle perpendicular to the artery was slowly introduced until blood reflux was obtained, when it was introduced slightly beyond until artery transfixation, confirmed by the lack of reflux. At this moment, recently prepared $30 \mathrm{~mL}$ of $1.6 \%$ lidocaine with 1:200000 epinephrine were slowly injected observing possible signs of vascular injection. Needle was then slowly backed, going once more through the artery lumen and being positioned anterior to it, when remaining $20 \mathrm{~mL}$ of the solution were injected. Needle was then removed and arm was positioned along the body with axillary compression. Five randomly selected patients received $1 \mathrm{~mL}$ iohexol contrast $\left(300 \mathrm{mg} \cdot \mathrm{mL}^{-1}\right.$ ) in each side of the artery before anesthetic injection for radiological evaluation.

Analgesia was evaluated in both groups by skin clamping and observing patients' pain manifestations, aiming at determining the extension of sensory block of musculocutaneous nerve in forearm radial border, radial nerve in dorsal face of hand, ulnar nerve in medial hand and fifth finger, and median nerve in the palm. The following parameters were evaluated: 1) anesthetic onset: time elapsed from beginning of puncture to loss of sensitivity in the four nerves; 2) sensory block duration: time elapsed between beginning of puncture and total sensory recovery; 3 ) motor block duration: time elapsed between beginning of puncture and total recovery of blocked limb; 4) tourniquet perception; and 5) complications and side effects.

Blockade was considered complete if all nerves were blocked with the preconized volume. If there was need for additional injection or anesthetic complementation, blockade was considered incomplete. In this case, additional anesthetic dose would only involve the nerve of the unblocked area. Blockade failure was defined as the need for general anesthesia. Lidocaine toxicity signs were investigated during anesthetic injection, such as hum, metal taste, heat sensation, visual disorders, dizziness or muscle contractions.

Tenoxicam (40 mg) and dipirone $\left(30 \mathrm{mg} \cdot \mathrm{kg}^{-1}\right)$ in $100 \mathrm{~mL}$ lactated Ringer's were administered at surgery completion and patients were transferred to the ward. All patients were followed up for 48 hours via telephone to check for complications on blockade site. If there were any signs, patient should return to the hospital to be evaluated by the authors. All patients were asked about their satisfaction with the technique. Results were evaluated by $t$ test with Welch correction for levels of freedom and by Fisher's exact test to compare two percentages, considering significant $p<0.05$.

\section{RESULTS}

Continuous variables results are shown as mean \pm standard deviation. Distribution by age, weight, height and gender is shown in table I; sensory and motor block onset and duration are shown in table II. Contrasted study of each nerve with the multiple stimulation technique is shown in figures 1,2 and 3, and with the transarterial technique in figures 4 and 5 .

Table I - Demographics Data

\begin{tabular}{|c|c|c|c|}
\hline & $\begin{array}{c}\text { MNS Group } \\
n=40\end{array}$ & $\begin{array}{c}\text { TA Group } \\
\mathrm{n}=30\end{array}$ & $p$ value \\
\hline Age (years) * & $38.6 \pm 12$ & $36.9 \pm 10.6$ & 0.53 \\
\hline Weight $(\mathrm{kg})^{*}$ & $67.7 \pm 11.4$ & $71.2 \pm 12.4$ & 0.13 \\
\hline Height $(\mathrm{cm})^{*}$ & $166.4 \pm 9.3$ & $168.6 \pm 10$ & 0.35 \\
\hline \multicolumn{4}{|l|}{ Gender } \\
\hline Male & 22 & 17 & \\
\hline Female & 18 & 13 & \\
\hline
\end{tabular}

* Values expressed in Mean \pm SD

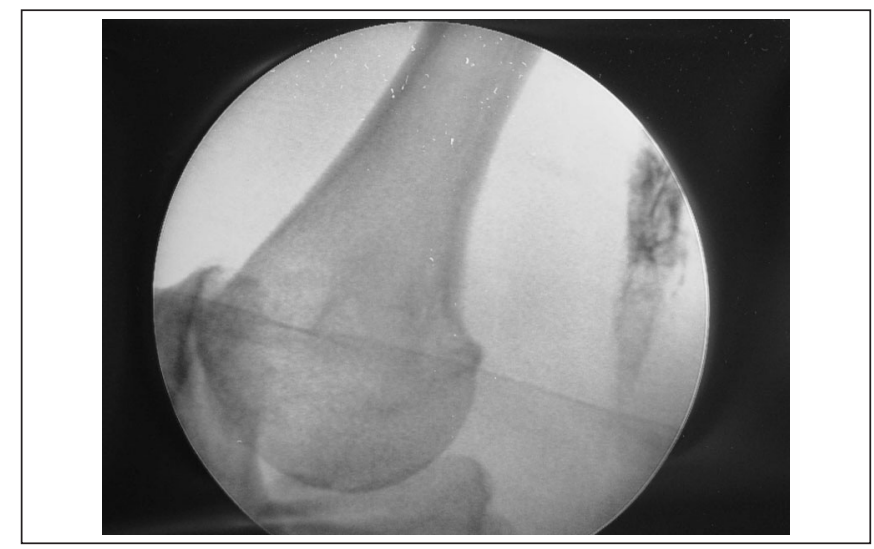

Figure 1 - Median Nerve Contrasted with Nerve Stimulator

Table II - Anesthetics Data

\begin{tabular}{|c|c|c|c|}
\hline & $\begin{array}{c}\text { MNS Group } \\
n=40\end{array}$ & $\begin{array}{c}\text { TA Group } \\
\mathrm{n}=30\end{array}$ & $p$ value \\
\hline Mean lidocaine dose $\left(\mathrm{mg} \cdot \mathrm{kg}^{-1}\right)$ & 13.5 & 12.5 & \\
\hline Maximum lidocaine dose $\left(\mathrm{mg} \cdot \mathrm{kg}^{-1}\right)$ & 16.6 & 15.09 & \\
\hline Onset (min) & $10.2 \pm 2.4$ & $8.8 \pm 2.3$ & 0.010 \\
\hline Sensory block duration (min) & $198.4 \pm 25.8$ & $184.3 \pm 26.1$ & 0.022 \\
\hline Motor block duration (min) & $201.4 \pm 20.2$ & $188 \pm 31.9$ & 0.038 \\
\hline
\end{tabular}




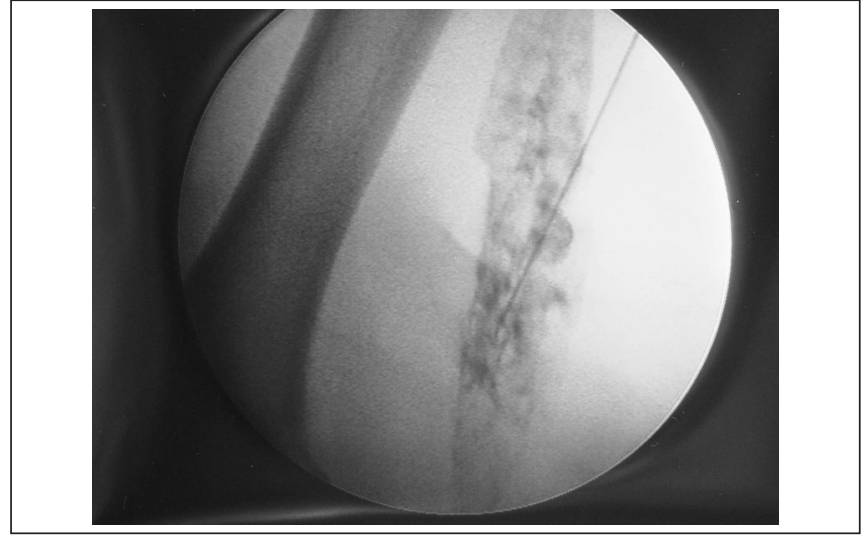

Figure 2 - Radial Nerve Contrasted with Nerve Stimulator

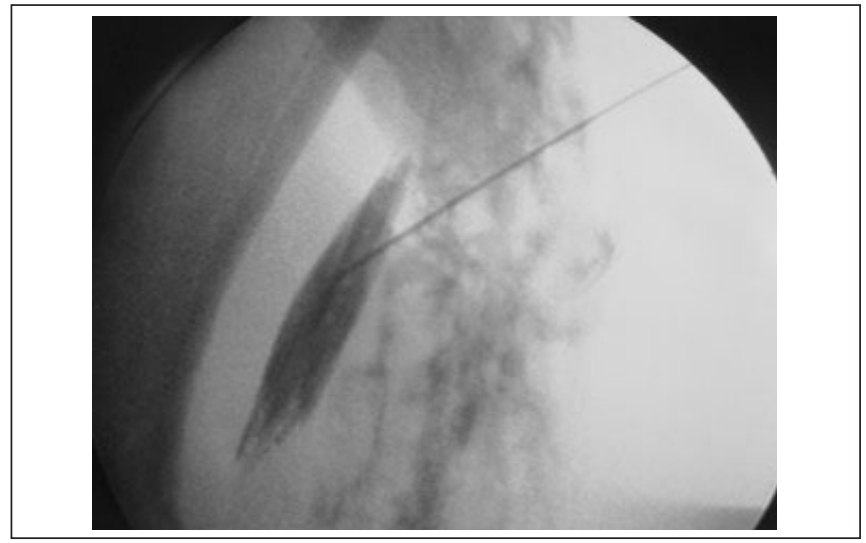

Figure 3 - Musculocutaneous Nerve Contrasted with Nerve Stimulator

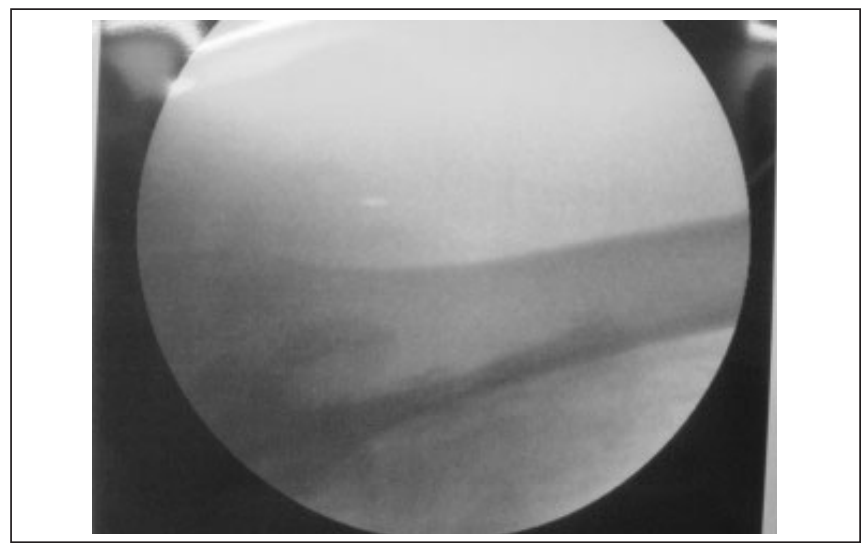

Figure 4 - Contrast by Injection Posterior to the Axillary Artery

Mean onset has been significantly shorter for the TA group. There has been no arterial or venous puncture with multiple stimulation technique.

Only two patients, both from the TA group, needed general anesthesia; remaining patients were operated with blockades. Artery was transfixed and anesthetic solution was de-

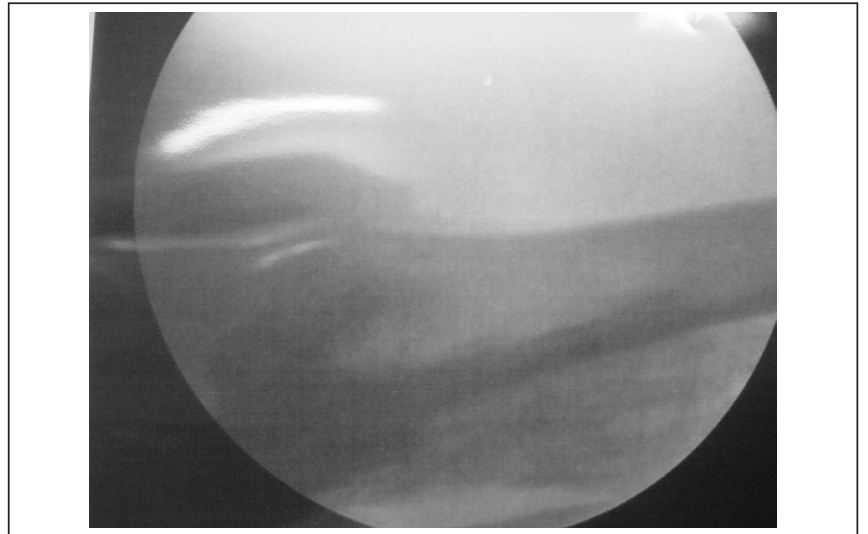

Figure 5 - Contrast by Injection Anterior to the Axillary Artery

posited posterior and anterior to it in all TA group patients. Muscle contraction in the territories innervated by median, radial or ulnar and musculocutaneous nerves was obtained in all MNS group patients and in three TA group patients. Musculocutaneous nerve was significantly easier to be blocked with the aid of nerve stimulator $(p=0.034)$ (Table III).

Table III - Blocked nerves, Nerves Complementation and Quality of Analgesia: Number of Patients in each Group

\begin{tabular}{lccc}
\hline & $\begin{array}{c}\text { MNS Group } \\
\mathrm{n}=40\end{array}$ & $\begin{array}{c}\text { TA Group } \\
\mathrm{n}=30\end{array}$ & p value \\
\hline Blocked nerves & 39 & 28 & 0.12 \\
$\quad$ Radial & 39 & 29 & 1.0 \\
Ulnar & 40 & 29 & 0.43 \\
Median & 39 & 25 & 0.034 \\
$\quad$ Musculocutaneous & & & 0.12 \\
Complementation of nerves & 1 & 0 & \\
$\quad$ Radial & 1 & 0 & \\
$\quad$ Ulnar & 0 & 0 & \\
$\quad$ Median & 1 & 3 & 0.68 \\
$\quad$ Musculocutaneous & $(92.5 \%)$ & $(83.3 \%)$ & \\
Quality of analgesia & 37 & 25 & \\
Adequate & 3 & 3 & \\
Partial & 0 & 2 & \\
Failure (general anesthesia) & &
\end{tabular}

Sensory and motor block duration was significantly longer for the MNS group as compared to the TA group.

Mean lidocaine dose was $13.5 \mathrm{mg} . \mathrm{kg}^{-1}$ for the MNS group being $16.6 \mathrm{mg} . \mathrm{kg}^{-1}$ the highest dose. Mean dose was 12.5 $\mathrm{mg} . \mathrm{kg}^{-1}$ for the TA group, with maximum dose of 15.09 $\mathrm{mg} \cdot \mathrm{kg}^{-1}$. There has been no clinical signs of systemic local anesthetic toxicity in both groups.

No patient has referred pain at surgery site. However, four patients have referred tourniquet pain, being two from the TA group and two from the MNS group, without significant difference. 
There were no nervous injuries in the MNS group or hematoma or anesthetic vascular injection in the TAgroup.

\section{DISCUSSION}

When several lidocaine plus epinephrine injections are axillary applied in the brachial plexus, the use of plexus stimulator ( 3 injections) results in a similar success rate to the transarterial technique (2 injections), however demanding longer time for surgery beginning. Several authors consider axillary block success when analgesia is present in all surgical areas. This definition is clinically similar success rates sufficient, but implies a false success rate as compared to different blockade techniques ${ }^{5}$. We have considered the blockade complete when all forearm nerves were blocked with the first injection. Complementation was needed in three MNS group patients in different nerves: musculocutaneous, radial and ulnar. Complementation was needed in three TA group patients and blockade failure was seen in two other patients of this same group.

The brachial plexus block requires continuous concern with the injected volume. The ratio between injected anesthetic volume and extension of analgesia was firstly described in $1961^{9}$. Although mean axillary neurovascular compartment volume is just $42 \mathrm{~mL}^{9}$, single 60 to $80 \mathrm{~mL}$ perivascular injection may result in partial block ${ }^{10,11}$. This was initially explained by insufficient proximal anesthetic spread to nervous branches and upper plexus divisions caused by the umeral head, but was not confirmed by the randomized controlled studies $^{12,13}$.

The two axillary block techniques improve anesthetic spread in the neurovascular sheath, as compared to single injection techniques: the transarterial with two injections and the multiple nerve stimulation with three or four injections. The transarterial technique with $800 \mathrm{mg}$ of $1.6 \%$ lidocaine with epinephrine has resulted in $95 \%$ to $100 \%$ success rate without any adverse effect ${ }^{1}$.

Anatomic studies have shown that musculocutaneous and axillary nerves leave neurovascular sheath at the coracoid process, in line with the fact that $50 \mathrm{~mL}$ of $1.6 \%$ lidocaine have not blocked the musculocutaneous nerve in three patients of this series. There are few studies on the multiple stimulation technique with nerve stimulator. A comparison of three axillary block techniques (catheter, paresthesia and nervous stimulation) has shown higher success rate when more than one nerve is stimulated ( $50 \%$ for $1,80 \%$ for 2 and $100 \%$ for 3 nervous stimulations) ${ }^{14}$. Three stimulations technique, where only median, radial and ulnar nerves are located, has just $50 \%$ success rate ${ }^{15}$. Our study has included isolated stimulation of musculocutaneous nerve, resulting in $92.5 \%$ success rate.

When anesthetic solution was injected close to 2 or 4 identified nerves, only 1 out of 15 patients needed complementation ${ }^{15}$. The comparison between single injection and injection after locating 3 nerves has resulted in $43 \%$ effectiveness versus $90 \%$, respectively ${ }^{4}$. It has been recently shown that double stimulation results in $92 \%$ success rate while single injection results in just $52 \%{ }^{16}$. Our study comparing triple injection after locating three nerves with the neurostimulator and the transarterial technique with two injections has resulted in $92.5 \%$ success rate with triple injection as compared to $83.3 \%$ with double injection.

Success rate depends on surgery site and blockade extension ${ }^{17}$ thus being hard to compare it among different studies. We were more concerned with analgesia spread than with motor block for surgical success. Initially we have not looked for the nerve innervating the surgical site, but rather for stimulation internal and external to the axillary artery. Median nerve is usually easier to be located with nerve stimulator and its investigation has become popular for the single injection method $^{17}$

Incomplete musculocutaneous nerve blockade is a common axillary technique problem because it is not close to the axillary artery ${ }^{18}$. In our study, double injection with the transarterial technique and the attempt to stimulate this nerve with the three stimulations technique has shown that this problem may be minimized with the latter approach.

Transarterial access is achieved with double injection, being one superficial and the other deep to the axillary artery. Success rate of $83.3 \%$ in our study was close to that observed in some studies $\left(79 \%{ }^{19}, 88.8 \%{ }^{20}\right)$, however lower than $92 \%{ }^{16}$ and $95 \%{ }^{1}$ of other studies. Success rate of $92.5 \%$ obtained with multiple stimulations is similar to results obtained by other studies, which have varied from $88 \%$ to $93 \%{ }^{4,5,8}$.

Notwithstanding the increased risk of complications such as vasospasm, hematoma and/or pseudoaneurysm 21,22, transarterial is considered a safe technique, shown by a prospective analysis of 1000 patients ${ }^{20}$. In our study, 48 hours follow up has not evidenced any complication. Major concern during multiple stimulation location is the possibility of puncture -induced neuropraxis. This possibility was minimized in our study by the nerve stimulator and by slow needle advance while current was decreased to $0.5 \mathrm{~mA}$. In addition, analgesic complementation was never injected in the same site of the previous injection.

Maximum lidocaine plus epinephrine dose recommended for regional anesthesia in adults is $7 \mathrm{mg} \cdot \mathrm{kg}^{-123}$. However, when lidocaine is diluted for infiltrative anesthesia, dose varies from $45 \mathrm{mg} \cdot \mathrm{kg}^{-1}$ in slim patients to $50 \mathrm{mg} \cdot \mathrm{kg}^{-1}$ in obese patients ${ }^{24}$. Lidocaine dose to obtain $5 \mu \mathrm{g} \cdot \mathrm{mL}^{-1}$ plasma concentration varies according to the administration site ${ }^{25}$.

There have been no signs of intoxication in 346 patients receiving $900 \mathrm{mg}$ lidocaine with epinephrine $(30 \mathrm{~mL}$ of $2 \%$ and $30 \mathrm{~mL}$ at $1 \%$ ) for transarterial brachial plexus block and success rate has been $100 \%{ }^{26}$. A different study has found mean plasma concentration of $2.9 \mu \mathrm{g} \cdot \mathrm{mL}^{-1}$ after transarterial brachial plexus block with $900 \mathrm{mg}$ lidocaine, with highest individual value of $5.6 \mu \mathrm{g} \cdot \mathrm{mL}^{-1}$ representing a dose of $18 \mathrm{mg} \cdot \mathrm{kg}^{-1}$ In this series, there has been no anxiety related to multiple injections technique because the method has been thoroughly explained, needle pathway has been anesthetized, stimulation was achieved with 0.5 to $1 \mathrm{~mA}$ and fentanyl has been previously administered. 
In conclusion, when axillary brachial plexus block is achieved with the aid of peripheral nerve stimulator and when the musculocutaneous nerve is stimulated together with radial and/or median and/or ulnar nerve, the result is similar to double injection transarterial technique, but with more effective musculocutaneous nerve block. However, time to surgery beginning is longer with the use of neurostimulation.

Transarterial dose of $900 \mathrm{mg}$ routinely used in more than 500 patients ${ }^{27}$ is $12.5 \%$ higher than $800 \mathrm{mg}$ used in this study. Highest dose has been $16.6 \mathrm{mg} \cdot \mathrm{kg}^{-1}$, lower than $18 \mathrm{mg} \cdot \mathrm{kg}^{-1}$ used in the above-mentioned study ${ }^{27}$. Similarly, there have been no clinical manifestations of systemic toxicity with lidocaine plus epinephrine.

\section{REFERÊNCIAS - REFERENCES}

01. Imbelloni LE, Pinto AL, Neumann CR - Bloqueio do plexo braquial pela via transarterial com altas doses de lidocaína 1,6\%. Rev Bras Anestesiol, 1989:39:273-276.

02. Oliva Filho AL - O bloqueio do plexo braquial via axilar. Rev Bras Anestesiol, 1995;45(Supl20):116-118.

03. Chaves IMM, Chaves LFM, Dias CL - Bloqueio do plexo braquial por via axilar com neuroestimulador: verificação da latência e da eficácia. Rev Bras Anestesiol, 2001;51:244-249.

04. Koscielniak-Nielsen ZJ, Stens-Pedersen HL, Knudesen Lippert F Readiness for surgery after axillary block: single or multiple injection techniques. Eur J Anaesthesiol, 1997;14:164-171.

05. Koscielniak-Nielsen ZJ, Hesselbjerg L, Fejlberg V - Comparison of transarterial and multiple nerve stimulation techniques for an initial axillary block by $45 \mathrm{ml}$ of mepivacaine $1 \%$ with adrenaline. Acta Anaesthesiol Scand, 1998;42:570-575.

06. Sia S, Bartoli M - Selective ulnar nerve localization is not essential for axillary brachial plexus block using a multiple nerve stimulation technique. Reg Anesth Pain Med, 2001;26:12-16.

07. Serradell Catalan A, Moncho Rodríguez JM, Santos Carnes JA et al - Anestesia de plexo braquial por via axilar. Cuántas respuestas buscamos com neuroestimulación? Rev Esp Anestesiol Reanim, 2001;48:356-363.

08. Koscielniak-Nielsen ZJ, Nielsen PR, Nielsen SL et al - Comparison of transarterial and multiple nerve stimulation techniques for axillary block using a high dose of mepivacaine with adrenaline. Acta Anaesthesiol Scand, 1999;43:398-404.

09. de Jong RH - Axillary block of the brachial plexus. Anesthesiology, 1961;22:215-225.

10. Vester-Andersen T, Christiansen $C$, Sorensen $M$ et al Perivascular axillary block. II: influence of injected volume of local anaesthetic on neural blockade. Acta Anaesthesiol Scand, 1983;27:95-98

11. Vester-Andersen T, Husum B, Lindeburg $T$ et al - Perivascular axillary block. IV: blockade following 40,50 or $60 \mathrm{ml}$ of mepivacaine $1 \%$ with adrenaline. Acta Anaesthesiol Scand, 1984:28:99-105.

12. Koscielniak-Nielsen ZJ, Horn A, Nielsen PR - Effect of arm position on the effectiveness of perivascular axillary block. Br J Anaesth, 1995; $74: 387-391$.

13. Koschielniak-Nielsen ZJ, Christensen LQ, Pedersen HL et al - Effect of digital pressure on the neurovascular sheath during perivascular axillary block. Br J Anaesth, 1995;75:702-706.

14. Baranowski AP, Pither CE - A comparison of three methods of axillary brachial plexus anaesthesia. Anaesthesia, 1990;45:362-365

15. Lavoie J, Martin R, Tetrault JP et al - Axillary plexus block using a peripheral nerve stimulator: single or multiple injections. Can $\mathrm{J}$ Anaesth, 1992;39:583-586

16. Inberg P, Annila I, Annila P - Double-injection method using peripheral nerve stimulator is superior to single injection in axillary plexus block. Reg Anesth Pain Med, 1999;24:509-513.

17. Brockway MS, Wildsmith JA - Axillary brachial plexus block: method of choice? Br J Anaesth, 1990;64:224-231.
18. Yamamoto K, Tsubokawa T, Shibata Ket al - Area of paresthesia as determinant of sensory block in axillary brachial plexus block. Reg Anesth, 1995;20:493-497.

19. Goldberg ME, Gregg C, Larijani GE et al - A comparison of three methods of axillary approach to brachial plexus blockade for upper extremity surgery. Anesthesiology, 1987;66:814-816.

20. Stan TC, Krantz MA, Solomon DL et al - The incidence of neurovascular complications following axillary brachial plexus block using a transarterial approach. A prospective study of 1,000 consecutive patients. Reg Anesth, 1995;20:486-492.

21. Merrill DG, Brodsky JB, Hentz RV - Vascular insufficiency following axillary block of the brachial plexus. Anesth Analg, 1981:60:162-164.

22. Zipkin M, Backus WW, Scott B et al - False aneurysm of the axillary artery following brachial plexus block. J Clin Anesth, 1991:3:143-145.

23. de Jong RH, Grazer FM - Perioperative management of cosmetic liposuction. Plast Reconstr Surg, 2001:107:1039-1044.

24. Klein JA - Anesthetic formulation of tumescent solutions. Dermatol Clin, 1999;17:751-759.

25. Tucker GT, Mather LE - Properties, Absorption and Disposition of Local Anaesthetics Drugs, em: Cousins MJ, Briedenbaugh PO Neural Blockade. Philadelphia, Lippincott, 1988;62-63.

26. Aantaa R, Kirvela O, Lahdenpera A - Transarterial brachial plexus anesthesia for hand surgery: a retrospective analysis of 346 cases. J Clin Anesth, 1994;6:189-192.

27. Palve $\mathrm{H}$, Kirvela $\mathrm{O}$, Olin $\mathrm{H}$ et al - Maximum recommended doses of lignocaine are not toxic. Br J Anaesth, 1995;74:704-705.

\section{RESUMEN}

Imbelloni LE, Beato L, Cordero JA - Comparación de las Técnicas Transarterial y de Estimulación de Múltiples Nervios para Bloqueo del Plexo Braquial por Vía Axilar usando Lidocaína con Epinefrina

JUSTIFICATIVA Y OBJETIVOS: La técnica transarterial con grandes dosis de anestésico local resulta en alta efectividad para el bloqueo axilar del plexo braquial. La técnica de utilizar múltiples estímulos exige más tiempo y mayor experiencia. Este estudio prospectivo compara la latencia y el índice de éxito del bloqueo del plexo braquial usando dos técnicas de localización: transarterial o múltipla estimulación de los nervios.

MÉTODO: La lidocaína con epinefrina, $800 \mathrm{mg}$, fue usada inicialmente para el bloqueo axilar. En el grupo transarterial, 30 $m L$ de lidocaína a $1,6 \%$ con epinefrina fueron inyectados profundamente y $20 \mathrm{~mL}$ superficialmente a la arteria axilar. En el grupo de múltipla estimulación, tres nervios fueron localizados eléctricamente y bloqueados con volúmenes 20 $m L, 20 \mathrm{~mL}$ y $10 \mathrm{~mL}$ de la solución. El bloqueo fue considerado efectivo cuando la analgesia estaba presente en todos los nervios en la área distal al codo.

RESULTADOS: El tiempo de latencia (8, $8 \pm 2,3$ min versus 10,2 $\pm 2,4$ min; $p$-valor $=0,010$ ) fue significativamente menor en el grupo transarterial. Bloqueos sensitivos completos en los cuatro nervios (mediano, ulnar, radial y musculocutáneo) fueron logrados en un $92,5 \%$ versus $83,3 \%$ en el grupo de múltipla estimulación y acceso transarterial, respectivamente sin diferencia significativa $(p$-valor $=0,68)$. El nervio musculocutáneo fue significativamente más fácil de bloquear con el estimulador de nervio periférico ( $p=0,034)$.

CONCLUSIONES: La técnica de múltipla estimulación para el bloqueo axilar usando estimulador de nervios (3 inyecciones) y la técnica transarterial (2 inyecciones) producen resultados semejantes en la calidad del bloqueo. El nervio musculocutáneo es más facilmente bloqueado con el uso del estimulador del nervio periférico. La técnica de múltipla estimulación necesitó menor suplementación del bloqueo y aumentó el tiempo para el inicio de la cirugía. 